\title{
Biplane opening wedge high tibial osteotomy with a distal tuberosity osteotomy, radiological and clinical analysis with minimum follow-up of 2 years
}

Juan Erquicia', Pablo Eduardo Gelber ${ }^{1,2}$, Simone Perelli ${ }^{1 *}$ (D), Federico Ibañez ${ }^{1}$, Maximiliano Ibañez ${ }^{1}$, Xavier Pelfort ${ }^{1,3}$ and Juan Carlos Monllau ${ }^{1,4}$

\begin{abstract}
Background: High tibial osteotomy is an established and helpful treatment for unicompatimental osteoarthritis associated with varus deformity. However, asupratubercle high tibial osteotomy leads to a decrease in patellar height making the technique not suitable in case of concomitant patella baja. Moreover, this kind of osteotomy can change in situ forces at patellofemoral joint and the lateral patellar tilt. With the aim to widen the indication of high tibial osteotomy was proposed a biplane opening wedge high tibial osteotomy with a distal tuberosity osteotomy (B-OWHTO). This technique provide that the tibial tuberosity remains joined to the tibial metaphysis so as not to theoretically alter the patellar height. However, some Authors claim that BOWHTO could lead to an increase in tibial slope. The purpose of the present study was to assess the tibial slope, patella-femoral changes and axial correction as well as functional outcomes following a B-OWHTO.
\end{abstract}

Methods: Patients operated on with a B-OWHTO and a minimum 24 months of follow-up were included. The mechanical alignment of the lower limb, patellar height, lateral patellar tilt and posterior tibial slope were calculated preoperatively, immediately after surgery and at the 24-month follow-up. The clinical results were evaluated using the Lysholm, Kujala and Hospital for Special Surgery knee scores. The possible postoperative development of patellofemoral pain or radiologic patellofemoral alteration was also evaluated.

Results: Twenty-three patients were included with a mean follow-up of 33 months (range 27-41). The mechanical alignment of the lower limb shifted from a mean $9.3^{\circ} \pm 2.5$ varus preoperatively to a mean $0.2^{\circ} \pm 2.2$ valgus postoperatively. No changes in patellar height, lateral patellar tilt or in the posterior tibial slope were observed. The mean Lysholm and HSS scores improved from $68.3 \pm 9.1$ and $64.2 \pm 5.2$ preoperatively to $93.2 \pm 2.1$ and $94.1 \pm 3.6$ at final follow-up $(p<0.01)$. The mean Kujala score improved from $67.3 \pm 9.8$ to $86.4 \pm 7.6$ at final follow up $(p<0.01)$. No patients developed both radiological or clinical symptoms at patellofemoral joint.

Conclusions: Open wedge high tibial osteotomy with a dihedral L-cut distal and posterior to the tibial tubercle accurately corrected axial malalignment without any change at patella-femoral joint or any modification to the posterior tibial slope while providing improved knee function at short-term follow-up. The radiographic as well as the clinical results support the use of this technique to treat medial compartment knee osteoarthritis and varus malalignment in young and middle-aged patients with a normal-to-low patellar height.

(Continued on next page)

\footnotetext{
* Correspondence: perelli.simone@gmail.com

'ICATME, Hospital Universitari Dexeus, UAB, Carrer de Sabino Arana, 5,

Barcelona 08028, Spain

Full list of author information is available at the end of the article
} 
(Continued from previous page)

Level of evidence: Case series with no comparison group, Level IV.

Keywords: High tibial osteotomy, Open wedge tibial osteotomy, Biplanar tibial osteotomy, Tuberosity osteotomy, Patellar height, Tibial slope

\section{Background}

High tibial osteotomy (HTO) is an established treatment for patients with medial compartment knee osteoarthritis (OA) and varus malalignment (Poignard et al. 2010). Open wedge high tibial osteotomy (OWHTO) has gained in popularity over recent years as a viable alternative to the traditional lateral closed wedge osteotomy. This technique has several advantages over the lateral closed wedge osteotomy. They include avoiding a fibular osteotomy and the risk of peroneal nerve complications (Gaasbeek et al. 2010), smaller surgical exposure without muscle detachment, easier and more precise correction that can be change even after the osteotomy cut (Bito et al. 2009; Hankemeier et al. 2010). Furthermore, it can make an eventual future knee replacement easier not only due to the location of the skin approach but more because an OWHTO decreases the metaphyseal deformity caused by a closed wedge (Hui et al. 2011).

However, OWHTO has some potential disadvantages for the patellofemoral joint. Firstly, because a supra-tubercle osteotomy can lower the height of the patella, which is particularly true when a large correction is required (Amzallag et al. 2013). As a consequence, the use of an OWHTO is not usually recommended in cases of patella infera. (Schallberger et al. 2011; Lobenhoffer et al. 2009) However, it should be kept in mind that the origin of patella infera is likely multifactorial and so even patients with a preoperative normal-to-low patellar height could end up with this condition. Additionally, OWHTO can increase patellofemoral contact pressure and cause patellofemoral degeneration overtime even when patellar height has not been modified. (Stoffel et al. 2007) For those reasons, some surgeons advise against OWHTO when patellofemoral pain or patellofemoral chondral alterations are present even in patients with normal patellar height (Il et al. 2017; Kim et al. 2016; Kloos et al. 2018). A study recently has shown that an alteration of the axial alignment of the patella with a change of the lateral patellar tilt can be observed with a standard OWHTO. This fact could contribute to explaining the changes described before (Lee et al. 2016).

A second possible drawback of supra-tubercle OWHTO is its tendency to increase the sagittal tibial slope (Akizuki et al. 2008). Increasing the tibial slope may affect antero-posterior translation of the tibia as well as the in-situ forces on the anterior cruciate ligament.

With the aim of solving these potential problems and so broaden OWHTO indications, some authors still support the use of the standard infra-tubercle HTO in these cases (Shim et al. 2013). However, moving the tibial osteotomy distally reduces the total surface of cancellous bone at the osteotomy site and increases the risk of non-union. Several authors have already reported superior clinical outcomes of traditional supra-tubercle OWHTO over infra -tubercle HTO due to lower non-union rates (Noyes et al. 2006).

The further solution suggested by some authors in previous studies to deal with these issues is to perform a modification of the standard technique: a biplane OWHTO with a distal tuberosity osteotomy (OWHTO-B). This technique provide that the tibial tuberosity remains joined to the tibial metaphysis so as not to theoretically alter the patellar height without increasing the patellofemoral pressions (28) or even diminishing them (Kloos et al. 2018).

The aim of this study was then to assess the tibial slope, any patello-femoral change and axial correction as well as functional outcomes following a OWHTO-B. It was hypothesized that while providing good clinical results, this technique would accurately correct the axial malalignment without producing patello-femoral radiological alterations and keeping the posterior tibial slope unchanged.

\section{Methods}

A retrospective analysis was performed of patients who had undergone an OWHTO-B for symptomatic medial osteoarthritis between 2012 and 2014 at the authors' institution. Patients who did not have the corresponding radiographies before surgery, immediately after surgery and 24 months after the index procedure were also excluded. All the radiographic studies had to be performed at the index institution.

Like for every tibial osteotomy, the general indications for surgery were the presence of a varus tibial deformity associated with symptomatic narrowing of the medial 
compartment space, the absence of a flexion contracture, a flexion range of motion of at least $90^{\circ}$ and the failure of other conservative treatment. OWHTO-B was particularly indicated when possibility of postoperative Caton Deschamp Index (CDI) less than 0.8 was present due to the risk of excessive and somehow unpredictable degree of patellar lowering: 1) in cases of a CDI less than $0.8,2$ ) in cases of CDI between 0.8 and 1 , but a planned correction greater than $10^{\circ}$ and, as extended indication, 3 ) in case of combined varus malalignment and patellofemoral pain or radiological patellofemoral alterations with a normal CDI. This is due to its ability to decrease the patellofemoral joint pressures (Kloos et al. 2018).

Contraindications were severe bone loss in the femoral condyle or tibial plateau, the presence of rheumatoid arthritis or infectious arthritis, the presence of prominent osteoarthritis in the lateral compartment and markedly limited joint motion.

The clinical research ethics committee of our institution approved the study (protocol number HTOL 2017-01). All the patients signed informed consent to participate in the study as well as for the evaluation and publication of their results.

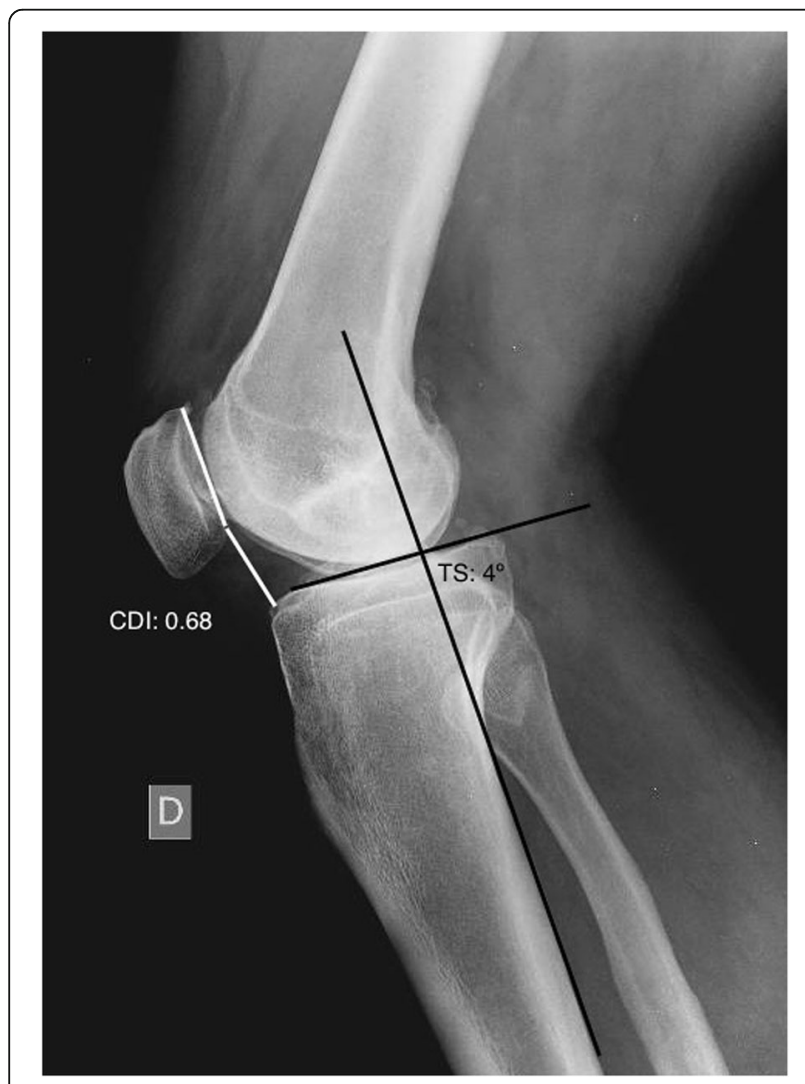

Fig. 1 Measurement of the Caton-Deschamps index and the posterior tibial slope. CDI: Caton- Deschamps index, TS: tibial slope

\section{Preoperative study}

- Full-weight-bearing long-leg standing anteroposterior radiographs were performed to determine the angle of the extremity and the desired degree of correction. A postoperative anatomic femoral-tibial axis of $5-8^{\circ}$ valgus and a mechanical axis around the Fujisawa point was the goal (Habata et al. 2006).

- The Schuss or Rosenberg radiographic view (Rosenberg et al. 1988) was used to evaluate the joint line space.

- A lateral non-weight-bearing radiography of the knee at $30^{\circ}$ of flexion was used to measure the posterior tibial slope and the patellar height. The angle of the posterior tibial slope was determined based on a line passing through the posterior cortex of the tibia and another parallel to the joint slope (Brazier et al. 1996). For patellar height, the CDI was measured as previously described (Caton et al. 1982) (Fig. 1).

- A Merchant view (skyline view with knee flexed to $45^{\circ}$ ) was used to calculate the lateral patellar tilt (LPT) and the severity of patellofemoral arthritis. The latter was classified into 4 stages according to Merchant et al. (Kim and Joo 2012) (Fig. 2)

Two observers, blind from the aim of the study, performed all the radiographic measurements. They were carried out using the PACS system (Centricity Enterprise Web V3.0; GE Healthcare).

\section{Surgical technique}

Two senior surgeons performed all the procedures. Once an arthroscopic evaluation of the knee had been done, a 6-7 cm-longitudinal approach mid-way between the tibial tuberosity (TT) and the posteromedial border of the tibia was performed. The semitendinosus and gracilis tendons were released and the superficial medial collateral ligament was freed from its distal insertion. The patellar tendon was identified and protected with a retractor.

\section{Osteotomy}

The OWHTO-B has two different osteotomy planes. While the horizontal cut is similar to the standard OWHTO, the vertical part of the osteotomy is performed posterior to the tibial tubercle. Under fluoroscopic control, one 2.4-mm guiding Kirschner wire was placed in the medial cortex of the tibia at the metaphyseal-diaphyseal transition zone with the aim of tipping the fibular head in a proximal and posterolateral direction. The K-wire guided the sagittal cut performed in the posterior two thirds of the tibia. It is crucial to maintain perfect perpendicularity to the main axis of the bone on the sagittal plane to avoid tibial slope 


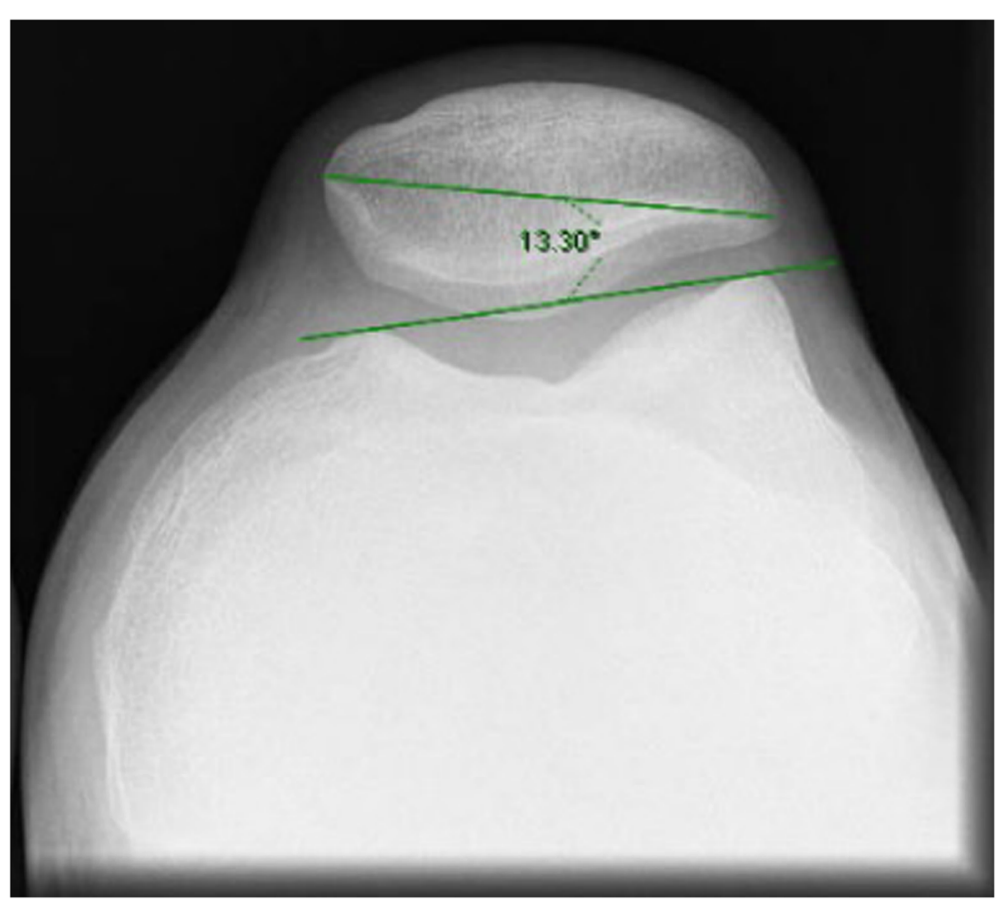

Fig. 2 Measurement of the Lateral Patellar Tilt

modifications. This section of the osteotomy ended 1 to $2 \mathrm{~cm}$ medial to the lateral border of the tibia. The second section of the osteotomy was vertical in the coronal plane and extended 3 to $4 \mathrm{~cm}$ distally. Thus, the TT together with the proximal segment of the osteotomized tibia was maintained. A thickness of $10 \mathrm{~mm}$ of the TT should be maintained in the most proximal part to minimize the risk of fracture. The desired correction is achieved using a metallic wedge introduced in the posterior-most part of the osteotomy site, thereby creating a trapezoidal gap to avoid an increase in the tibial slope (Fig. 3). To prevent anterior tilting of the TT leading to an increase in the posterior tibial slope, one or two anteroposterior cortical screws fixing the TT were secured before the osteotomy plate was placed. Then, the locking LOQTEQ ${ }^{\circ} \mathrm{HTO}$ plate (Berlin, Germany), was accordingly fixed (Fig. 4). Finally, the osteotomy gap was filled with an iliac crest allograft.

\section{Rehabilitation protocol}

Patients started continuous passive motion of the knee as well as isometric quadriceps strengthening exercises immediately after surgery. During the first 3 weeks, patients were only allowed toe-touch partial weight-bearing. Then, progressive weight bearing as tolerated started. Full weight bearing was allowed after week 6 .

Complications were recorded during the study period, which had a minimum follow-up of 24 months.
The same preoperative radiological studies were carried out at the latest follow up.

\section{Clinical assessment}

Clinical and functional follow-up included the Lysholm, Kujala and Hospital for Special Surgery (HSS) knee scores. The physical examination as well as the functional evaluation of every patient was performed preoperatively and at final follow-up by a single sports medicine surgeon who was independent of the study. Attention was paid to the clinical evaluation of any postoperative patellofemoral pain manifested by patients.

\section{Radiological follow up}

In every case a standard X-ray protocol was obtained preoperative and at 1-6-12-24 months postoperative. The protocol comprised a full-weight-bearing long-leg standing anteroposterior radiographs, a lateral non-weightbearing radiography of the knee at $30^{\circ}$ of flexion, a Merchant view. Femoral-tibial angle, posterior tibial slope, CDI, LPT and degree of patellofemoral arthritic degeneration were collected. Immediately postoperative was not obviously possible to obtain a full-weight-bearing long-leg standing X-ray as well as a Merchant view. Only standard anteroposterior and lateral non-weight-bearing radiography of the knee at $30^{\circ}$ of flexion were obtained.

CDI and posterior tibial slope were collected starting from the immediate postoperative radiographs. 

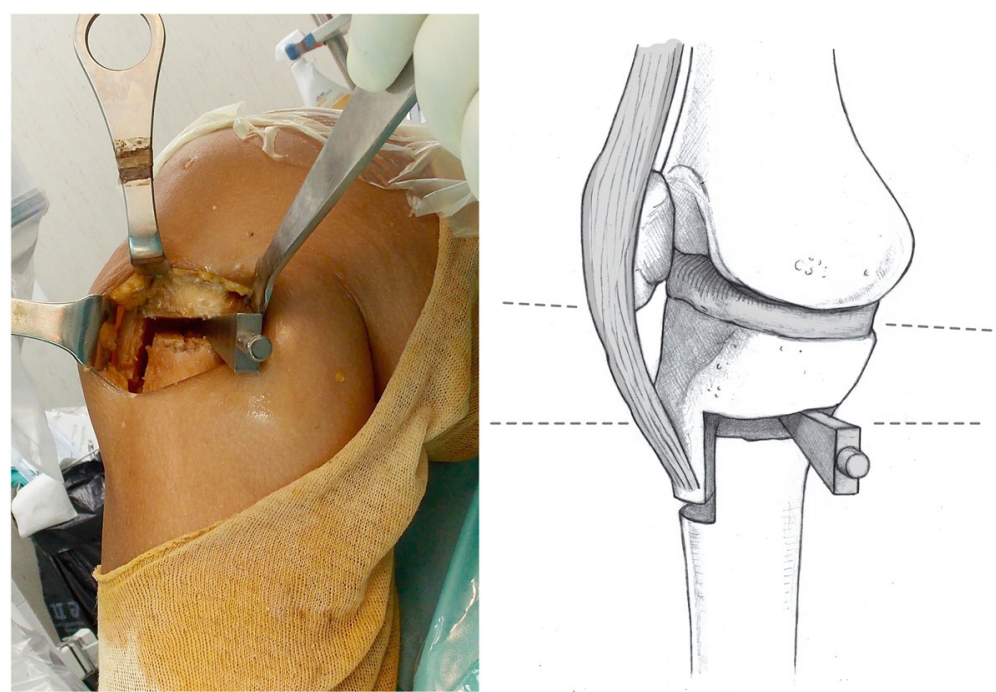

Fig. 3 Graduated wedge at the level of the posterior tibial cortex

LPT, femoral-tibial angle and severity of patella-femoral arthritis were collected starting from the 1-month follow-up radiographs.

\section{Statistical analysis}

Continuous variables are presented as means, standard deviations (SD), maximums and minimums. Categorical variables are presented as percentages and frequencies.

For analysis of the repeated measures, Bonferroni's correction was used to identify differences between baseline and follow-up radiographic measurements.

Because of the small sample number, statistical tests were not utilized to evaluate normality. Instead, the assessment was performed with non-parametric equivalents, which showed no discrepancies in terms of significance. The inference in continuous variables was calculated with the paired-samples T-test and their results are presented with their 95\% confidence interval (95\% CI). Interobserver variability was analyzed with the variance test. The level of significance was set at $5 \%(\alpha=$ $0.05)$, a bilateral approximation. All the analyses were performed with the SPSS 19 (SPSS Inc., Chicago, Illinois).

\section{Results}

During the studied period 33 L-OWHTO were performed. Ten patients were excluded because some of the necessary postoperative images were lacking. Twenty-three patients (15 males and 8 females) with a mean age of 42.7 years (range 20.1 to 54.3 years) were finally included. The median body mass index was 27.4 (range of 21.6-32.7). The


Fig. 4 Postoperative aspect, without significant modifications in patellar height or the posterior tibial slope 
mean follow up was 33 months (range 27-41) Concomitant surgical procedures were: 1 anterior cruciate ligament reconstruction (4.3\%), 8 meniscectomy (34.7\%), 4 microfractures at medial condyle (17.4\%).

\section{Radiological assessment}

The mechanical alignment of the lower limb shifted from a mean varus of $9.3^{\circ} \pm 2.5$ preoperatively to a mean valgus of $0.2^{\circ} \pm 2.2$ four weeks after surgery and to a mean valgus of $0.4^{\circ} \pm 2.6$ at the 24-month follow-up assessment (Table $1)$. The osteotomy did not alter neither the posterior tibial slope nor the patellar height in the immediate postoperative radiography (Table 1 and Figs. 5 and 6). No differences were noted neither in lateral patellar tilt at 1-month postoperative X-ray (Table 1). All these parameters remained unchanged at the 24-month follow-up (Table 1). Radiographically, most of the patellofemoral joints (20 out of 23) were graded I or II in the Merchant stage system. Merchant grades of the patellofemoral joints were not significantly different at preoperative evaluation and at latest follow up. There were no differences in the measures calculated for the two observers.

\section{Clinical results}

The mean Lysholm and HSS scores improved from 68.3 \pm 9.1 and $64.2 \pm 5.2$ preoperatively to $93.2 \pm 2.1$ and 94.1 \pm 3.6 at final follow-up $(p<0.01)$. The mean Kujala score improved from $67.3 \pm 9.8$ to $86.4 \pm 7.6$ at final follow up $(\mathrm{p}<0.01)$. Preoperatively 5 out of 23 patients reported mild patellofemoral pain, at the final follow up no patients complained about anterior knee pain.

Only 2 out of the 23 patients included in the study showed complications. One patient had a pulmonary thromboembolism. This patient had had similar episode two decades before. It was successfully treated with oral anticoagulants. Another patient required hardware removal 28 months after surgery. No problems relative to unions or infections were observed.

\section{Discussion}

The most important finding of the present study was that a OWHTO-B accurately corrected varus axial malalignment in patients with mid-to-low patellar height without causing any patellofemoral change or increasing the posterior tibial slope. The patients also had improved outcomes in the evaluated functional outcomes. All these findings confirmed our hypothesis. The rate of complication was low and all the osteotomies healed without delayed union.

Traditional OWHTO techniques cause distalization and lateralization of the TT, resulting in a decrease of patellar height as suggested by Goutallier using a mathematical model (Goutallier et al. 1979). In a recent study, Amzallag et al. showed that patellar height decreased more than $20 \%$ in one-third of patients after an OWHTO. (Amzallag et al. 2013)

These changes would negatively affect the functional results of the osteotomy. Likewise, several recent studies confirmed the advantages of the closed-wedge HTO over the OWHTO in relation to patellar height preservation (Bin et al. 2016; Gaasbeek et al. 2004; El-Amrani et al. 2010; Ozel et al. 2015; Brinkman et al. 2008; Noyes et al. 2005; Stoffel et al. 2007; Ferner et al. 2018). This is even more important in higher axial deviations as a correlation between the degree of axial correction and the lowering of the patella has been shown (Amzallag et al. 2013). With the OWHTO-B technique, the tibial tuberosity remains attached to the proximal fragment of the osteotomy. Subsequently, the patellar height remained unchanged. The OWHTO-B is not intended to increase patellar height as it does not move the patella upwards. Therefore, this technique is not recommended in cases of a Caton Deschamp Index less than 0.6. Instead, a combined HTO and a separate osteotomy of the TT to heighten the tuberosity is indicated to handle the varus deformity and the patellar height.

Other authors have already shown some similar modifications to the traditional OWHTO technique in order to avoid modifications of patellar height. Gaasbeek et al. (Gaasbeek et al. 2004), in a study with 17 patients, showed the results of an OWHTO preserving the TT attached to the proximal tibia and also fixing the TT with an anteroposterior screw. However, the authors did so after the osteotomy plate was fixed. Then, this anteroposterior screw could not have prevented any eventual anterior tilting of the proximal fragment. In addition, the

Table 1 Statistical analyses between preoperative and final follow-up results

\begin{tabular}{lllllll}
\hline & $\begin{array}{l}\text { Preoperative } \\
\text { mean score } \pm \text { SD }\end{array}$ & $\begin{array}{l}\text { Postoperative } \\
\text { mean score } \pm \text { SD }\end{array}$ & $\begin{array}{l}24 \text { months } \\
\text { postoperative } \\
\text { mean score } \pm \text { SD }\end{array}$ & $\begin{array}{l}\text { Comparison of } \\
\text { preop and postop } \\
(P \text { value })\end{array}$ & $\begin{array}{l}\text { Comparison of preop } \\
\text { and 24 months postop } \\
(P \text { value })\end{array}$ & $\begin{array}{l}\text { Comparison of postop } \\
\text { and 24 months postop } \\
(P \text { value })\end{array}$ \\
\hline Mechanical axis & $9.2^{\circ} \pm 2.5^{\circ}$ & $-0.2^{\circ} \pm 2.2^{\circ}$ & $-0.4^{\circ} \pm 2.6^{\circ}$ & $<0.001$ & $<0.001$ & NS \\
Patellar height & $0.94 \pm 0.1$ & $0.95 \pm 0.08$ & $0.95 \pm 0.12$ & NS & NS & NS \\
Tibial slope & $4.4^{\circ} \pm 2.1^{\circ}$ & $5.1^{\circ} \pm 1.4^{\circ}$ & $5.2^{\circ} \pm 1.4^{\circ}$ & NS & NS & NS \\
Lateral Patellar Tilt & $13.1^{\circ} \pm 2.9^{\circ}$ & $12.9^{\circ} \pm 3.2^{\circ}$ & $12.8^{\circ} \pm 3.7^{\circ}$ & NS & NS & NS \\
\hline
\end{tabular}




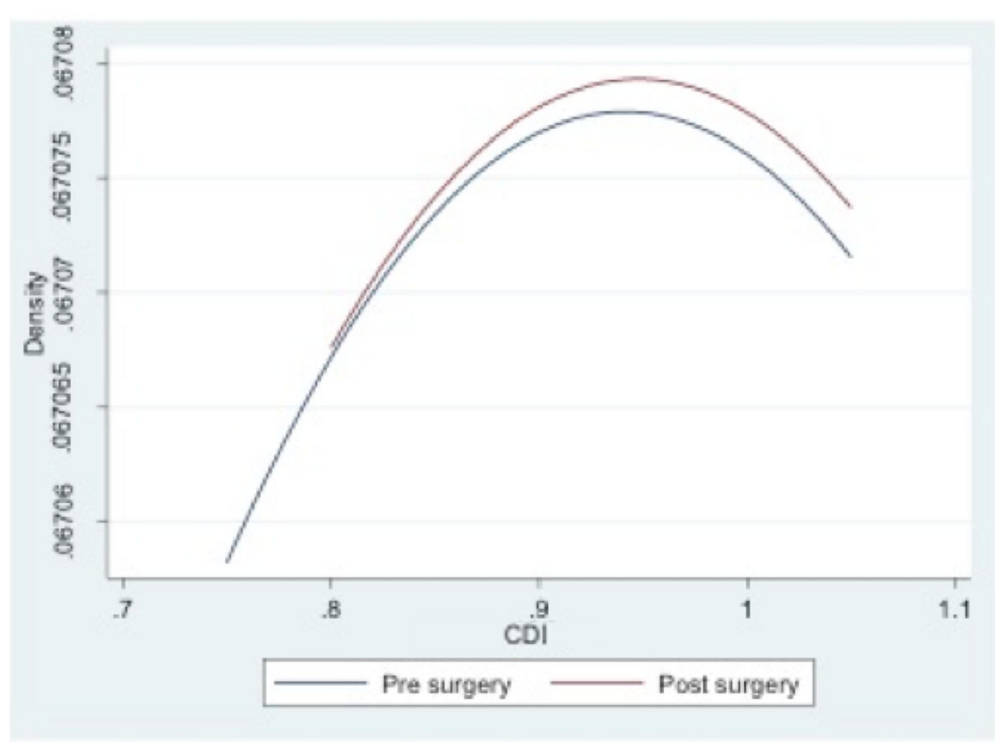

Fig. 5 Pre- and postoperative Caton-Deschamps indexes

authors did not measure the tibial slope changes. They also had two complications. One of the patients had a fracture of the TT and another patient had a delayed union. Conversely, no complications related to the technique were observed in the current study. It is likely that the difference in the sequencing of the osteotomy's fixation provided better contact between the tibial tuberosity and the distal tibial fragment.

To avoid modifications in patellar height and the tibial slope, Shim et al. (Shim et al. 2013) analyzed the results of an OWHTO distal to the TT. Despite achieving acceptable clinical and radiological results and because of the possibility of delayed union, they suggested the use of this technique only for those patients with an open physis and/or require minor corrections, have a body mass index below 25 and that the osteotomy gap be filled with autogenous iliac crest bone graft.

Standard OWHTO is contraindicated in patients with patella infera. The Caton-Deschamps index defines patella baja as those between 0.6 and 0.8 . Those below 0.6 are considered a patella infera. However, there is no consensus on its threshold in terms of deciding whether to perform the OWHTO as the procedure itself decreases patellar height. In a retrospective level IV evidence study



Fig. 6 Pre- and postoperative tibial slopes 
of patients operated on with an OWHTO, El Amrani et al. (El-Amrani et al. 2010) observed that the worse functional results were associated with postoperative patella infera. Gaasbeek et al. (Gaasbeek et al. 2004) also found a significant reduction in the CDI after OWHTO. All the patients showed lowering of the patella after the osteotomy and a direct correlation was found between the degree of wedge opening and the decrease in patellar height. In the present study, the OWHTO-B was performed when possibility of postoperative CDI less than 0.8 was present due to the risk of excessive and somehow unpredictable degree of patellar lowering.

Kim et al., using an SPET/TC evaluation, have shown that an increased signal activity around the patellofemoral joint was present after an OWHTO even when a low decrease of postoperative Blackburne-Peel ratio was found (Kim et al. 2016). Kim et al. reported that 21.9\% of the patients developed patellar OA and $41.2 \%$ developed a trochlear OA after OWHTO. Their findings were based on a second look arthroscopy made at 21 to 32 months of follow-up (Il et al. 2017). The prominent finding was that no correlation of this evolution with patellar height was found. The conclusion could be that even when the patellar height has not been changed, alterations in patellofemoral pressure forces happen after a OWHTO. That is also supported by a recent cadaveric biomechanical study (Kloos et al. 2018). In any case we must take into account that always a large deformity correction in medial open-wedge high tibial osteotomy may cause a degeneration of patellofemoral cartilage. (Otakara et al. 2019) in the present study an arthroscopic second look wasn't provide, but Kim in his study showed as well that $11.4 \%$ of the cases had postoperative anterior knee pain and among them all showed progressed OA on second look arthroscopy. Postoperative anterior knee pain was related to the ICRS grade of the patellofemoral joint at the time of second-look arthroscopy. In our study no patients complained about patellofemoral pain at the last follow up, neither the patients who reported patello-femoral discomfort before surgery. Finally, in their OWHTO series, Lee et al. have shown postoperative statistically significant changes in lateral patellar tilt (Lee et al. 2016). Even if they reported that this data has no clinical impact at 2 years follow-up, this mechanical alteration could as well explain patellofemoral cartilage subclinical alteration and patellofemoral pain at a longer term follow up. In the present study no significant alterations in postoperative lateral patellar tilt was detected at minimum 24 months follow up.

Several studies have shown that HTO might result in tibial slope changes. (Lu et al. 2018; Wu et al. 2017) in general, the posterior tibial slope increases after open-wedge high tibial osteotomies and decreases after the closed-wedge procedures. The increase in the tibial slope might alter in situ cruciate ligament forces and influence knee stability (Ozel et al. 2015). Changes to the tibial slope might also accelerate the process of joint degeneration due to alterations in knee kinematics and biomechanics as it leads to higher tibiofemoral contact pressure on the anterior portion of the tibial plateau and decreased pressure on the posterior femoral condyle (Brinkman et al. 2008).

To maintain the posterior slope, it is suggested that the anterior opening of the osteotomy site should be half of the posterior opening of the wedge (Noyes et al. 2005). The current technique, on the other hand, avoids any increment in the tibial slope by creating a trapezoidal gap and first fixing the TT with an anteroposterior screw.

The main limitation of this study was the low number of patients and the lack of control group. However, it is the largest reported series on such technique. Another limitation is that the short follow-up did not allow for the assessment long-term functional and radiological outcomes. Notwithstanding, the purpose of the investigation was to rule out any immediate radiological flaws of this relatively new technique.

For that reason, we excluded patients with an incomplete postoperative radiographic evaluation. However, the latter could be considered as a selection BIAS.

\section{Conclusions}

Biplane OWHTO with a distal tuberosity osteotomy accurately corrected axial malalignment without changing patellar femoral height, lateral patellar tilt or the posterior tibial slope while providing improved knee function at short-term follow-up.

\section{Abbreviations}

CDI: Caton Deschamp Index; HSS: Hospital for Special Surgery; HTO: High tibial osteotomy; LPT: Lateral patellar tilt; OA: Osteoarthritis; OWHTO: Open wedge high tibial osteotomy; OWHTO-B: Biplane opening wedge high tibial osteotomy with a distal tuberosity osteotomy; SD: Standard deviation;

Tा: Tibial tubercle

\section{Acknowledgements}

We would like to thank Mr. Eric L. Goode for his help with the English correction and Mr. Felip A. Montobbio for his work in the illustration process.

\section{Authors' contributions}

All authors read and approved the final manuscript.

\section{Ethics approval and consent to participate}

All procedures performed in the present study were in accordance with the ethical standards of the institutional research committee and with the 1964 Helsinki declaration and its later amendments or comparable ethical standards.

\section{Consent for publication}

Informed consent was obtained from all individual participants included in the study.

\section{Competing interests}

On behalf of all authors, the corresponding author states that there is no conflict of interest. 


\section{Publisher's Note}

Springer Nature remains neutral with regard to jurisdictional claims in published maps and institutional affiliations.

\section{Author details}

'ICATME, Hospital Universitari Dexeus, UAB, Carrer de Sabino Arana, 5, Barcelona 08028, Spain. ${ }^{2}$ Department of Orthopaedic Surgery, Hospital de la Sta Creu i Sant Pau, UAB, Carrer de Sant Quintí, 89, Barcelona 08026, Spain. ${ }^{3}$ Department of Orthopaedic Surgery, Consorci Sanitari de I'Anoia, Hospital de Igualada, Av. Catalunya, 11, Barcelona, Spain. ${ }^{4}$ Department of Orthopaedic Surgery, Hospital del Mar. Universitat Autònoma de Barcelona (UAB), Passeig Marítim, 25, Barcelona 08003, Spain.

Received: 2 December 2018 Accepted: 12 February 2019

Published online: 28 February 2019

\section{References}

Akizuki S, Shibakawa A, Horiuchi H, Yamazaki I, Horiuchi H (2008) The long-term outcome of high tibial osteotomy: a ten- to 20-year follow-up. J Bone Joint Surg Br 90:592-596

Amzallag J, Pujol N, Maqdes A, Beaufils P, Judet T, Catonne Y (2013) Patellar height modification after high tibial osteotomy by either medial openingwedge or lateral closing-wedge osteotomies. Knee Surg Sports Traumatol Arthrosc 21:255-259

Bin SI, Kim HJ, Ahn HS, Rim DS, Lee DH (2016) Changes in patellar height after opening wedge and closing wedge high tibial osteotomy: a meta-analysis. Arthroscopy 32:2393-2400

Bito H, Takeuchi R, Kumagai K, Aratake M, Saito I, Hayashi R, Sasaki Y, Aota Y, Saito T (2009) A predictive factor for acquiring an ideal lower limb realignment after opening wedge high tibial osteotomy. Knee Surg Sports Traumatol Arthrosc 17:382-389

Brazier J, Migaud H, Gougeon F, Cotton A, Fontaine C, Duquennoy A (1996) Evaluation of methods for radiographic measurement of the tibial slope: a study of 83 healthy knees. Rev Chir Orthop 3:195-200

Brinkman JM, Lobenhoffer P, Agneskirchner JD, Staubli AE, Wymenga AB, van Heerwaarden RJ (2008) Osteotomies around the knee: patient selection, stability of fixation and bone healing in high tibial osteotomies. J Bone Joint Surg Br 90:1548-1557

Caton J, Deschamps G, Chambat P, Lerat JL, Dejour H (1982) Patella infera: apropos of 128 cases. Rev Chir Orthop Reparatrice Appar Mot 68:317-325

El-Amrani MH, Lévy B, Scharycki S, Asselineau A (2010) Patellar height relevance in opening-wedge high tibial osteotomy. Orthop Traumatol Surg Res 96:3743

Ferner F, Lutter C, Dickschas J, Strecker W (2018) Medial open wedge vs. lateral closed wedge high tibial osteotomy - indications based on the findings of patellar height, leg length, torsional correction and clinical outcome in one hundred cases. Int Orthop [Epub ahead of print]

Gaasbeek RD, Nicolaas L, Rijnberg WJ, van Loon CJ, van Kampen A (2010) Correction accuracy and collateral laxity in open versus closed wedge high tibial osteotomy. A one-year randomized controlled study. Int Orthop 34: 201-207

Gaasbeek RD, Sonneveld H, van Heerwaarden RJ, Jacobs WCH, Wymenga AB (2004) Distal tuberosity osteotomy in open wedge high tibial osteotomy can prevent patella infera: a new technique. Knee 11:457-461

Goutallier D, Delepine G, Debeyre J (1979) The patella-femoral joint in osteoarthritis of the knee with genu varus. Rev Chir Orthop Reparatrice Appar Mot 65:25-31

Habata T, Uematsu K, Hattori K, Kasanami R, Takakura Y, Fujisawa Y (2006) High tibial osteotomy that does not cause recurrence of varus deformity for medial gonarthrosis. Knee Surg Sports Traumatol Arthrosc 14:962-967

Hankemeier S, Mommsen P, Krettek C, Jagodzinski M, Brand J, Meyer C, Meller R (2010) Accuracy of high tibial osteotomy: comparison between open- and closed- wedge technique. Knee Surg Sports Traumatol Arthrosc 18:13281333

Hui C, Salmon LJ, Kok A (2011) Long-term survival of high tibial osteotomy for medial compartment osteoarthritis of the knee. Am J Sports Med 39:64-70

II KK, Kim DK, Song SJ, Lee SH, Bae DK (2017) Medial open-wedge high Tibial osteotomy may adversely affect the patellofemoral joint. Arthrosc J Arthrosc Relat Surg 33:811-816
Kim TW, Kim BK, Kim DW, Sim JA, Lee BK, Lee YS (2016) The SPECT/CT evaluation of compartmental Changesafter open wedge high Tibial osteotomy. Knee Surg Relat Res 28:263-269

Kim YM, Joo YB (2012) Patellofemoral osteoarthritis. Knee Surg Relat Res 24:193200

Kloos F, Becher C, Fleischer B, Feucht MJ, Hohloch L, Südkamp N, Niemeyer P, Bode G. (2018) High tibial osteotomy increases patellofemoral pressure if adverted proximal, while open-wedge HTO with distal biplanar osteotomy discharges the patellofemoral joint: different open-wedge high tibial osteotomies compared to an extra-articular unloading device. Knee Surg Sports Traumatol Arthrosc. (Epub, ahed of print). https://doi.org/10.1007/ s00167-018-5194-X

Lee YS, Lee SB, Oh WS, Kwon YE, Lee BK (2016) Changes in patellofemoral alignment do not cause clinical impact after open-wedge high tibial osteotomy. Knee Surg Sports Traumatol Arthrosc 24:129-133

Lobenhoffer P, Van Heerwaarden R, Staubli A, Jakob R (2009) Osteotomies around the knee. In: AO Fundation publishing

Lu J, Tang S, Wang Y et al (2018) Clinical outcomes of closing- and openingwedge high Tibial osteotomy for treatment of anteromedial Unicompartmental knee osteoarthritis. J Knee Surg [Epub ahead of print]

Noyes FR, Goebel SX, Wfest J (2005) Opening wedge tibial osteotomy: the 3triangle method to correct axial alignment and tibial slope. Am J Sports Med 33:378-387

Noyes FR, Mayfield W, Barber Westin SD, Albright JC, Heckmann TP (2006) Opening wedge high tibial osteotomy: an operative technique and rehabilitation program to decrease complications and promote early union and function. Am J Sports Med 34:1262-1273

Otakara E, Nakagawa S, Arai Y et al (2019) Large deformity correction in medial open-wedge high tibial osteotomy may cause degeneration of patellofemoral cartilage: a retrospective study. Medicine (Baltimore) 98:14299

Ozel O, Yucel B, Mutlu S, Orman O, Mutlu H (2015) Changes in posterior tibial slope angle in patients undergoing open-wedge high tibial osteotomy for varus gonarthrosis. Knee Surg Sports Traumatol Arthrosc Mar 13. [Epubahead of print]

Poignard A, Flouzat Lachaniette CH, Amzallag J, Hernigou P (2010) Revisiting high tibial osteotomy: fifty years of experience with the opening-wedge technique. J Bone Joint Surg Am 92:187-195

Rosenberg TD, Paulos LE, Parker RD, Coward DB, Scott SM (1988) The forty-fivedegree posteroanterior flexion weight-bearing radiograph of the knee. J Bone Joint Surg Am 70:1479-1483

Schallberger A, Jacobi M, Wahl P, Maestretti G, Jakob RP (2011) High tibial valgus osteotomy in unicompartmental osteoarthritis of the knee: a retrospective follow-up study over 13-21 years. Knee Surg Sports Traumatol Arthrosc 19: 122-127

Shim JS, Lee SH, Jung HJ, Lee HI (2013) High tibial open wedge osteotomy below the tibial tubercle: clinical and radiographic results. Knee Surg Sports Traumatol Arthrosc 21:57-63

Stoffel K, Willers C, Korshid O, Kuster M (2007) Patellofemoral contact pressure following high tibial osteotomy: a cadaveric study. Knee Surg Sports Traumatol Arthrosc 15:1094-1100

Wu L, Lin J, Jin Z et al (2017) Comparison of clinical and radiological outcomes between opening-wedge and closing-wedge high tibial osteotomy: a comprehensive meta-analysis. PLoS One 9(12):e0171700

\section{Submit your manuscript to a SpringerOpen ${ }^{\circ}$ journal and benefit from:}

- Convenient online submission

- Rigorous peer review

- Open access: articles freely available online

- High visibility within the field

- Retaining the copyright to your article

Submit your next manuscript at $>$ springeropen.com 